\title{
A Importância da Consulta de Enfermagem em Pré-operatório de Ostomias Intestinais
}

\author{
The Importance of the Nursing Visit Prior to Intestinal Ostomy Placement
}

Regiane de Souza Mendonça ${ }^{1}$, Marcus Valadão², Leonaldson Castro ${ }^{3}$, Teresa Caldas Camargo ${ }^{4}$

\section{Resumo}

No cuidado aos pacientes portadores de ostomias intestinais, observa-se, no período pós-operatório, a ocorrência de dificuldades em aceitar a nova condição de ostomizado, e o deficit de conhecimentos sobre o cuidado com o estoma (autocuidado). A partir de tais observaçōes, surge a necessidade da consulta de enfermagem no préoperatório de ostomias, no sentido de: possibilitar ao paciente melhor aceitação da ostomia e do seu tratamento; orientá-lo sobre o procedimento a ser realizado; estimular o autocuidado e prevenir complicações comuns no local de inserção da ostomia. O objetivo desse trabalho é destacar a importância da consulta de enfermagem nesse cenário, bem como descrever os aspectos a serem abordados para se atingir um cuidado integral e humanizado. A metodologia utilizada para elaborar esta sistematização constou de pesquisa bibliográfica, seguida de leitura analítica do material coletado e utilização da teoria do autocuidado e do deficit de autocuidado de Orem, como suporte teórico. Espera-se, desta forma, que a utilização da consulta de enfermagem no pré-operatório de ostomias sirva de instrumento capaz de melhorar a qualidade de vida desses pacientes e possibilitar o aprendizado para o autocuidado eficiente.

Palavras-chave: Ostomia, Cuidado de enfermagem, Autocuidado

\footnotetext{
${ }^{1}$ Serviço de Quimioterapia - Oncotech Oncologia, Rio de Janeiro (RJ), Brasil

${ }^{2}$ Serviço de Emergência do Hospital do Câncer II - Instituto Nacional de Câncer (INCA), Rio de Janeiro (RJ), Brasil

${ }^{3}$ Serviço de Cirurgia Abdominopélvica do Instituto Nacional de Câncer (INCA), Rio de Janeiro (RJ), Brasil

${ }^{4}$ Hospital do Câncer III - Instituto Nacional de Câncer (INCA), Rio de Janeiro (RJ), Brasil

Endereço para correspondência: Regiane de Souza Mendonça. Rua Paissandú, 385/ 302 - Flamengo, Rio de Janeiro (RJ), Brasil - CEP: 22210-080.

E-mail: regianemendonca@yahoo.com.br
} 


\section{INTRODUÇÃO}

As intervenções cirúrgicas que resultam em ostomias intestinais são muito comuns em oncologia, trauma e cirurgia gastroenterológica, o que por si justificaria o tema em foco, não fosse, também, a constatação da inquietude dos pacientes ostomizados demonstrada durante seguimento pós-operatório.

Estes pacientes, ao serem questionados sobre seu tratamento no cotidiano assistencial, mostram dificuldade em compreender a cirurgia realizada e suas conseqüências. $\mathrm{O}$ deficit de conhecimento se expressa quando estes pacientes observam em seu abdome uma parte do intestino exteriorizada, mostrando dificuldade em aceitar sua nova condição. Portanto, fica clara a necessidade de se estabelecer um canal de comunicação e empatia com o paciente no pré-operatório, considerando as alterações físicas e emocionais conseqüentes à cirurgia. A avaliação pré-operatória de uma cirurgia geradora de estoma é imprescindível para que se alcance uma reabilitação eficiente voltada para o autocuidado e reduzam-se suas taxas de complicaçōes ${ }^{1}$.

A fase pré-operatória é uma etapa muito importante, pois o paciente se encontra abalado pelas informaçóes acerca de sua doença, da intervenção cirúrgica e da construção de um estoma. Acrescente-se ainda que a assistência ao paciente candidato à confecção de estoma deve ser planejada, sistematizada e individualizada ${ }^{2}$.

Diante do exposto, refletiu-se sobre a importância de um serviço sistematizado de enfermagem em nível ambulatorial, voltado especificamente para o cuidado de pacientes em fase pré-operatória, candidatos à ostomia intestinal, a fim de orientá-los sobre o processo cirúrgico que envolve a internação hospitalar, o preparo para a cirurgia, sua recuperação e os cuidados que necessitarão adotar no pós-operatório. Neste sentido, optou-se por construir um roteiro sistematizado da consulta de enfermagem para pacientes portadores de neoplasia intestinal em pré-operatório de ostomias intestinais, com a intenção de que esta intervenção possibilite a redução da tensão, da desinformação e estimule o autocuidado, facilitando a reabilitação no pós-operatório e a melhor aceitação do tratamento.

A consulta de enfermagem é, além de uma ação de ajuda, uma ação de aprendizado na qual enfermeiro e paciente interagem, buscando solucionar problemas identificados por meio do diagnóstico de enfermagem ${ }^{3}$.

A consulta sistematizada visa a: orientar o paciente portador de neoplasia intestinal no período préoperatório de inserção da ostomia, possibilitando uma melhor compreensão da ostomia e do seu tratamento; demarcar o local ideal onde será confeccionada a ostomia; estimular o paciente para o autocuidado e com isso prevenir complicações comuns no local de inserção da ostomia.

O objetivo desse trabalho é destacar a importância da sistematização na consulta de enfermagem em préoperatório de ostomias intestinais, bem como descrever os aspectos a serem abordados e avaliados na consulta para se atingir um cuidado integral e humanizado.

\section{METODOLOGIA}

A constatação do deficit de conhecimentos sobre o procedimento cirúrgico e os cuidados no pós-operatório com a ostomia geraram a necessidade de uma sistematização da consulta de enfermagem no préoperatório de ostomias intestinais, cuja elaboração constou das seguintes etapas: (1) Pesquisa bibliográfica, utilizando como fontes artigos nacionais e internacionais e livros. A busca foi feita com as palavras-chave: ostomias e câncer intestinal; (2) Leitura analítica do material resultante da pesquisa bibliográfica com sua posterior organização; (3) Elaboração da proposta de sistematização da consulta de enfermagem no préoperatório de ostomias intestinais; (4) Utilização da teoria do autocuidado e do deficit de autocuidado de Orem, para dar suporte teórico à sistematização.

\section{CUIDADOS NO PRÉ-OPERATÓRIO DE OSTOMIAS}

A palavra estoma tem origem grega a partir do étimo stóma, que exprime a idéia de boca e tem como sinônimo estômato. Considera-se ostomia ou estoma, toda e qualquer abertura cirúrgica de uma víscera oca ao meio externo, direta ou indiretamente $e^{4}$

As ostomias intestinais são consideradas definitivas se o procedimento visa a substituir a função do ânus. Nos casos de tratamento cirúrgico do câncer de reto, a ressecção abdominoperineal leva à construção de um ostomia definitiva, obrigatoriamente, em conseqüência da remoção do reto e do ânus. Estas geralmente são terminais, sendo o segmento colônico seccionado 5 .

A visão de que o ostomizado necessitava muito mais que uma boa e efetiva técnica cirúrgica para a sua reabilitação, como empatia, informações e instruções personalizadas, levou Rupert Turnbull a convidar uma de suas pacientes, Norma Gill para auxiliá-lo nessa atividade. Em 1958, Turnbull a contratou para atuar como "técnica em ostomia", e assim nascia a estomaterapia, com Norma Gill sendo considerada a primeira estomaterapeuta, e Turbull o pai de estomaterapia. A estomaterapia é hoje uma especialidade estabelecida da enfermagem e o seu especialista é denominado estomaterapeuta $(\mathrm{ET})^{6}$. 
A fase pré-operatória das ostomias deve priorizar a avaliação do paciente nas esferas física e psicossocial, identificando o nível de autocuidado prévio e em vigência da doença ${ }^{7}$. Nesta fase tanto o paciente como os seus familiares estão ávidos e receptivos por informaçôes que lhes dêem subsídios para trabalhar a ansiedade e o medo do desconhecido e ativar os mecanismos de enfrentamento ${ }^{1}$.

As variáveis que devem ser avaliadas durante a consulta pré-operatória são o conhecimento do indivíduo sobre o seu diagnóstico e possibilidades de tratamento; os antecedentes familiares relacionados ao diagnóstico, buscando um perfil epidemiológico; os antecedentes alérgicos principalmente de pele, os hábitos de eliminação e respectivas alteraçôes promovidas pela doença e tratamento; as atividades de vida diária relacionadas ao autocuidado; as atividades sociais, de lazer e trabalho; o estado emocional, identificando o impacto da doença e as expectativas quanto ao estoma, além de avaliar o nível de ansiedade e as estratégias de enfrentamento; o padrão cultural e respectivas influências étnicas e religiosas, observando-se o nível de compreensão, o estado nutricional, as habilidades psicomotoras, as condiçóes da parede abdominal e o aspecto da região perineal ${ }^{1}$.

A presença da ostomia, como resultado da medida terapêutica utilizada, determina alteraçóes profundas na pessoa, as quais se expressam por desajustes físicos, psicoemocionais e sociais que, se não forem trabalhados, irão influenciar na efetividade do processo de reabilitação ${ }^{8}$.

A educação é de importância vital, particularmente em relação ao autocuidado com as ostomias. Orientando corretamente sobre a técnica de troca da bolsa coletora, assim como sobre a observação do estoma durante a troca, a enfermeira vai ajudar a prevenir complicações e tornar a vida com a ostomia mais manejável?

A determinação pré-operatória da posição do estoma possibilita a aderência e a permanência do dispositivo coletor por um período maior (3-6 dias), o que facilita a participação social e a redução de custos e, assim, contribuir para melhorar a qualidade de vida da pessoa ostomizada ${ }^{10}$.

\section{TEORIA DO AUTOCUIDADO DE OREM}

Durante a construção deste trabalho, buscou-se uma teoria que fundamentasse os objetivos propostos nesta sistematização. Assim, optou-se por adotar a Teoria do autocuidado e do deficit de autocuidado de Orem por entender que estas pessoas são portadoras de doença crônica (câncer) e se tornarão portadores de ostomia intestinal. Portanto, é necessário promover um processo educacional para que estes se tornem aptos a cuidar de si.

Autocuidado na visão de Orem é o conjunto de açôes que o indivíduo realiza para seu próprio benefício, na intenção de manter sua saúde e bem-estar. O autocuidado, quando efetivo, contribui para o desenvolvimento humano e, quando há seu impedimento ou limitação, ocorre o deficit do autocuidado que indica a necessidade de atuação da enfermagem ${ }^{11}$.

A teoria de deficit de autocuidado constitui a essência da teoria geral de enfermagem de Orem. Ela identifica cinco métodos de ajuda, sendo: agir ou fazer para o outro; guiar o outro; apoiar o outro; proporcionar um ambiente que promova o desenvolvimento pessoal, tornando-se capaz de satisfazer demandas futuras ou atuais de ação; e ensinar ao outro.

Assim, a teoria de enfermagem de Orem oferece uma base abrangente para a prática da enfermagem, incluindo a educação permanente como parte do componente profissional da educação em enfermagem. Sua premissa de autocuidado é contemporânea dos conceitos de promoção e manutenção da saúde. $\mathrm{O}$ autocuidado na teoria de Orem é comparável à saúde holística, pois ambas promovem a responsabilidade do indivíduo pelo cuidado da saúde. Isto é especialmente relevante na atualidade, visto que a alta hospitalar vem sendo antecipada, aumentando a demanda de cuidados em casa e dos serviços ambulatoriais ${ }^{12}$.

\section{Proposta de sistematização da CONSUlta de ENFERMAGEM NO PRÉ-OPERATÓRIO DE OSTOMIAS INTESTINAIS}

$O$ processo de enfermagem aplicado na consulta em estomaterapia, de acordo com a teoria de Orem compreende: avaliação, diagnóstico de enfermagem, plano, implementação e evolução.

\section{Avaliação}

$\mathrm{Na}$ fase de avaliação, o enfermeiro coleta dados referentes ao estado de saúde do paciente; perspectivas quanto à sua saúde; metas de saúde no contexto do histórico de vida, estilo de vida e estado de saúde; exigências de autocuidado; e capacidade do paciente para efetuar o autocuidado. São também coletados dados acerca dos conhecimentos, habilidades, motivação e orientação do paciente.

Para se obter a história do paciente em pré-operatório de ostomia intestinal é necessária uma breve consulta ao seu prontuário, buscando conhecer a doença de base, tratamentos prévios (cirurgias, quimioterapia ou radioterapia), planejamento terapêutico, outras doenças associadas, uso de medicamentos e condição social.

A entrevista com o paciente e sua família é primordial, ou melhor, é o ponto forte que caracteriza a consulta de enfermagem, na qual se pretende estabelecer um relacionamento interpessoal, sentimento de confiança e respeito num ambiente agradável, 
tranqüilo e confidencial.

$\mathrm{O}$ exame físico do paciente que será submetido a uma cirurgia que envolve ostomia intestinal deve ser completo, a fim de buscar qualquer deficiência e/ou anormalidade que poderá afetar o cuidado do estoma.

\section{Diagnóstico de Enfermagem}

O diagnóstico de enfermagem será baseado nos deficits de autocuidado identificados na avaliação do paciente.

\section{Plano}

O plano deve ser coerente com o diagnóstico, baseado nas demandas de autocuidado identificadas, sempre promovendo o paciente e sua família como agentes de cuidado.

$\mathrm{Na}$ consulta em pré-operatório de ostomias intestinais, o enfermeiro deve utilizar alguns dos métodos de ajuda identificados por Orem que propiciam a realização do autocuidado, sendo a orientação, o apoio, o ensino e propiciar um ambiente que promova o desenvolvimento pessoal.

As orientações devem esclarecer sobre o que é uma ostomia intestinal, para que serve, como se comporta, como cuidar, como será a vida diária e social, evidenciando os benefícios do tratamento.

Mostrar fotos de estomas intestinais com e sem bolsa coletora, permitir que o paciente e sua família manipulem os materiais que farão uso, permitir o contato direto e real com o assunto e uma prévia do que irá acontecer, prevenindo surpresas no pósoperatório imediato.

A orientação quanto aos cuidados de pré-operatório deve englobar ainda a dieta adequada de acordo com o protocolo do serviço, o preparo colônico que será realizado quando internado, o jejum na noite anterior à cirurgia e os cuidados gerais de pré-operatório.

\section{Implementação}

A implementação do plano visa a promover o paciente como agente de autocuidado, satisfazer as necessidades de autocuidado e diminuir os deficits de autocuidado. Nessa fase do processo será realizada a demarcação do local do estoma.

Está comprovado que o estoma bem localizado na parede abdominal facilita as atividades de autocuidado referentes à remoção e à colocação da bolsa, à higiene do estoma e pele periestoma, bem como a manutenção do sistema coletor, contribuindo para prevenir complicaçōes, possibilitar a reintegração social precoce, constituindo, ainda, um direito do paciente. A demarcação do local do estoma deve ser realizada sempre que possível em conjunto com o cirurgião, a fim de melhor discutir o planejamento da incisão e a proposta do estoma, sendo importante a participação do paciente no processo de demarcação.

\section{Método para Marcar 0 LOCAL da INSERÇ̃̃o dO ESTOMA}

- Verificar o tipo de estoma a ser realizado: este fator possibilita saber o segmento do intestino a ser exteriorizado para determinar o quadrante abdominal onde será localizado o estoma;

- Localizar o músculo reto abdominal;

- Escolher o local do estoma conforme os seguintes requisitos: abaixo da margem costal; planejar o local da incisão; distanciar quando possível de antigas cicatrizes, pregas cutâneas, linha da cintura, crista ilíaca e cicatriz umbilical (Figura 1);

- Marcar claramente o local com uma caneta dermográfica;

- Quando em dúvida - marcar duas localizações;

- Solicitar ao paciente que se sente, levante e deite para observar o local demarcado nas diferentes posiçóes;

- Verificar a margem de fixação dos dispositivos que deve ter uma área de $4 \mathrm{~cm}^{2}$ a $5 \mathrm{~cm}^{2}$ em relação ao local demarcado;

- Quando necessários dois estomas (urostomia e colostomia, ou ileostomia), estes não devem estar localizados no mesmo nível, devido ao possível uso da cinta;

- Atentar para atividades no trabalho, lazer e prática de esportes;

- Na ileostomia em alça ou terminal, o estoma deve ser localizado no quadrante inferior direito;

- Na colostomia de cólon descendente ou sigmóide, o estoma deve ser localizado no quadrante inferior esquerdo.

\section{Evolução}

A evolução do paciente será avaliada ao longo do processo, quando este apresentará respostas em relação ao seu tratamento, demonstrada através da eficácia das ações da enfermagem.

\section{CONCLUSÃO}

$\mathrm{Na}$ prática diária, os cuidados de enfermagem às pessoas portadoras de ostomia intestinal pretendem estabelecer uma relação efetiva de cuidado, uma reflexão sobre essa vivência e a maneira pela qual é possível contribuir para melhorar a assistência, facilitando a reabilitação e estimulando o autocuidado eficiente. Neste sentido, a assistência de enfermagem ao paciente que irá se submeter à cirurgia geradora de ostomia deve englobar, além das orientaçôes gerais relativas ao 


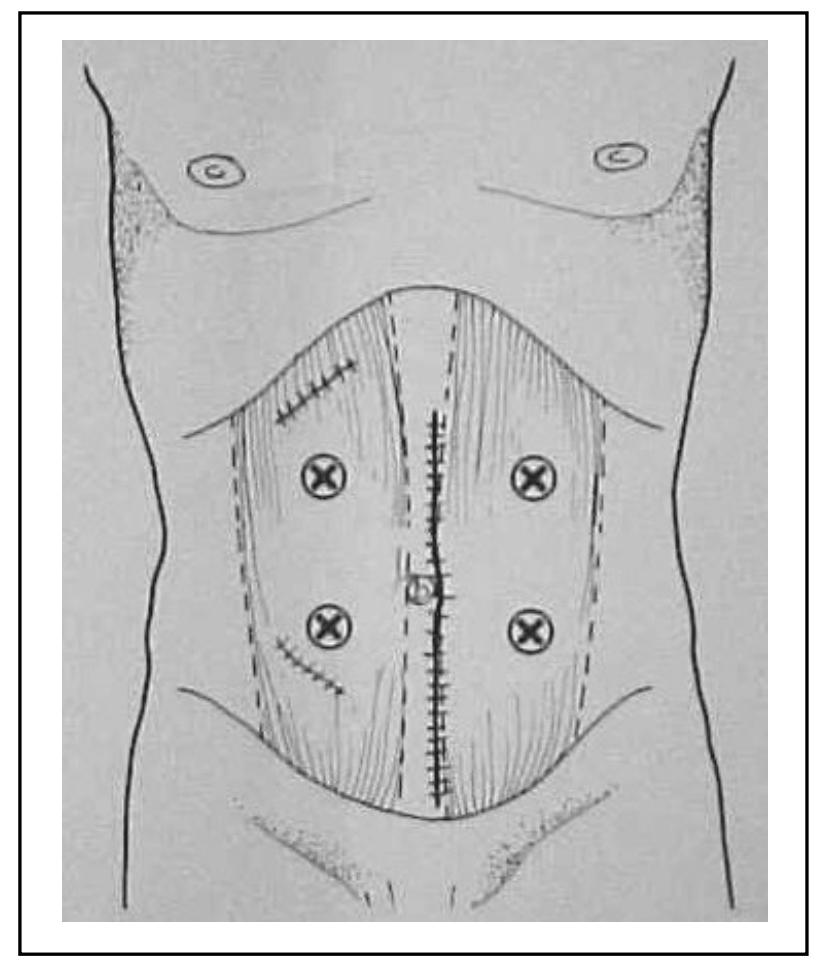

Figura 1. Localização de estomas no músculo reto abdominal

tratamento cirúrgico e suas conseqüências, ações específicas para o autocuidado, que devem ser planejadas e executadas em todas as fases do tratamento.

Além disso, por meio da consulta de enfermagem no pré-operatório de ostomias intestinais, a enfermeira fornece as orientações em relação aos cuidados com a ostomia, como o cuidado com a pele periestomal e a troca da bolsa coletora, e ainda demarca o local onde será inserida a ostomia. Com essa sistematização, esperase contribuir para a redução das complicaçōes no pósoperatório e/ou permitir que os pacientes as identifiquem precocemente. Ademais, há o intuito de prestar uma assistência de enfermagem humana e singular, voltada para as necessidades dos pacientes e melhoria da sua qualidade de vida.

\section{REFERÊNCIAS}

1. Cezaretti IUR, Santos VLCG, Filippin MJ, Lima SRS. O cuidar de enfermagem na trajetória do ostomizado: pré \& trans \& pós-operatórios. In: Santos VLCG, Cesaretti IUR (eds). Assistência em estomoterapia: cuidando do ostomizado. São Paulo: Atheneu; 2000:113-31.

2. Barroso UA, Ligneul G. Programa de treinamento para equipe de enfermagem: assistência de enfermagem em estomaterapia nas pacientes portadoras de neoplasia ginecológica. Rio de Janeiro: INCA; 2000;2-12.

3. Vanzin AS, Nery MES. Consulta de enfermagem: uma necessidade social? 2a ed. Porto Alegre: RM\&L; 2000.

4. Habr-Gama A, Araújo SEA. Estomas intestinais: aspectos conceituais e técnicos. In: Santos VLCG, Cesaretti IUR (eds). Assistência em estomoterapia: cuidando do ostomizado. São Paulo: Atheneu; 2000:39-54.

5. Carvalho FA, Wantanabe LM, Mendelssonh P. Colostomias. In: Pohl FF, Petroianu A. Tubos, sondas e drenos. Rio de Janeiro: Guanabara Koogan; 2000.

6. Santos VLCG. Estomaterapia através dos tempos. In: Santos VLCG, Cesaretti IUR (eds). Assistência em estomoterapia: cuidando do ostomizado. São Paulo: Atheneu; 2000:1-17.

7. Santos VLCG. Fundamentos teórico-metodológicos da assistência aos ostomizados na área da saúde do adulto. Rev Esc Enf USP. 2001;34(1):59-63.

8. Cezareti IUR. Ostomizado: reabilitação sem fronteiras? Ponto de vista do enfermeiro. Acta Paul Enf. 1995;8(1):11-17.

9. Shultz JM. Preparing the patient for colostomy care: a lesson well learned. Ostomy Wound Manage. 2002;10:22-25.

10. Meirelles CA, Ferraz CA. Avaliação da qualidade do processo de demarcação do estoma intestinal e das intercorrências tardias em paciente ostomizado. Rev Lat Am Enf. 2001;28:32-38.

11. Foster PC, Bennett AM. Dorothea E. Orem. In: George JB. Teorias de enfermagem: os fundamentos da prática profissional. Cap 7. 4a ed. Porto Alegre: Artmed; 2000.

12. Foster PC, Janssens NP. Dorothea E. Orem. In: George JB. Teorias de enfermagem: os fundamentos da prática profissional. Cap 7. Porto Alegre: Artmed; 1993.

\section{Abstract}

Care of intestinal ostomy patients has shown that they frequently experience difficulties in accepting their new condition and lack knowledge about stoma care (or self-care). This situation emphasizes the importance of the preoperative nursing visit, that is, prior to intestinal ostomy placement, in order to encourage patients' acceptance of the ostomy, provide information about the surgical procedure, encourage patients' self-care, and prevent common complications related to stoma placement. This article discusses the importance of the nursing visit in this setting and describes essential aspects for humanized, comprehensive care. The methodology used to elaborate this systematization consisted of a literature review followed by critical analysis. The self-care theory and Orem's selfcare deficit theory were used as the theoretical basis. The aim is for the preoperative nursing visit to help improve patients' quality of life by orienting them in efficient self-care.

Key words: Ostomy, Nursing care, Self-care 